\title{
Factors Associated with Gastrointestinal Bleeding During Hospitalization for Transcatheter Aortic Valve Replacement: A Retrospective Cohort Study
}

\section{Ted Andrew Spiewak ( $\square$ taspiewak@ucdavis.edu )}

UC Davis Medical Center https://orcid.org/0000-0002-6990-7781

\section{Armen Eskandari}

University of California Davis

\section{Tim W. Wang}

University of California Davis

\section{Heejung Bang}

University of California Davis

\section{Benjamin R. Stripe}

University of California Davis

Jeong W. Choi

University of California Davis

Garrett B. Wong

University of California Davis

Jeffrey A. Southard

University of California Davis

Walter D. Boyd

University of California Davis

\section{Sooraj Tejaswi}

University of California Davis

\section{Research article}

Keywords: Anemia, Anticoagulants, Antiplatelet therapy, Aortic stenosis, Gastrointestinal bleeding,

Transcatheter aortic valve replacement

Posted Date: July 10th, 2019

DOI: https://doi.org/10.21203/rs.2.11207/v1

License: (1) (1) This work is licensed under a Creative Commons Attribution 4.0 International License. Read Full License 


\section{Abstract}

Background \& Aims Gastrointestinal bleeding (GIB) is the most common bleeding complication post transcatheter aortic valve replacement (TAVR). Yet, the literature is sparse and incomplete. This study aims to identify the incidence and risk factors for GIB immediately following TAVR. Methods A retrospective study was performed among 489 patients who were hospitalized for TAVR at UC Davis Medical Center from (January 1, 2012 to June 30, 2017). The incidence of both upper and lower GIB, and potential risk factors during the same hospitalization were reviewed. Univariate analysis was done to identify independent risk factors. Results Mean age was 81.6 years and $49 \%$ were male, $23 \%$ had CKD3 or worse, $34 \%$ had history of prior GIB. Mean baseline Hgb and platelet count were $12.0 \mathrm{~g} / \mathrm{dl}$ and $197000 / \mathrm{ml}$, respectively. Dual antiplatelet therapy and anticoagulation were used in $396(81 \%)$ and $155(32 \%)$ patients, respectively. Gastric prophylaxis with either PPIs or $\mathrm{H} 2$ receptor blockers was used in $336(69 \%)$. Incidence of GIB was $1.4 \%$ (7/489) with 5 cases of upper GIB, 1 small intestinal GIB and 1 lower GIB. The most common culprit lesion found during endoscopy was angiodysplasia (3/5) and successful endoscopic intervention was performed. On univariate regression analysis, only a low hemoglobin level on admission was associated with increased likelihood of GIB (OR=1.49, $p=0.051$ per $1 \mathrm{~g} / \mathrm{dl}$ decrease). Conclusions GIB is rare immediately post-TAVR. Angiodysplasia was the most common etiology in our series. Baseline anemia is a predictor of GIB in this setting.

\section{Background}

Aortic stenosis (AS) is the most prevalent valvular disease in the United States with increasing prevalence as advances in health care increase the life expectancy of the US population. The prevalence and severity of AS increases with age, with 1 in 8 people older than age 75 showing moderate to severe AS [1-3].

Since the FDA approval of the Edwards SAPIEN device in November 2011, rates of transcatheter aortic valve replacement (TAVR) have increased significantly from 4627 procedures in 2012 to 34892 procedures in 2016 [4]. With introduction of new technology and expansion of its indications, TAVR is only expected to grow in the coming years [5]. Gastrointestinal bleeding (GIB) has been shown to be the most common bleeding complication post TAVR, likely due to a multitude of factors such as advanced age, use of antiplatelet agents and anticoagulants, chronic kidney disease, and a prior history of GI pathology [6, 7].

Gastroenterologists are commonly consulted out of concern for GIB in patient hospitalized after TAVR. However, there are not many studies that address the rate and patterns of GIB during index hospitalization. In the present paper, we review a large series of patients in a single tertiary care hospital to characterize the patterns of both upper and lower GIB in the index hospitalization for TAVR and identify potential risk factors.

\section{Methods}

\section{Study Design and Patient Population}


This is a retrospective cross-sectional study of all consecutive patients with symptomatic severe AS who were hospitalized for TAVR at UC David Medical Center (UCDMC) from January 1, 2012 to June 30, 2017. TAVR procedures were performed via either the transapical or transfemoral approach. Sapien 3 Valve (Edwards Lifesciences) and Medtronic CoreValve Evolut R (Medtronic) were used in most patients. Following TAVR, most patients were treated with single or dual antiplatelet agents such as aspirin or P2Y12 inhibitors; however, there were variations in this regimen due to patients requiring anticoagulation for reasons such as venous thromboembolism, atrial fibrillation, etc. A loading dose of P2Y12 inhibitor was administered before TAVR in patients who were not taking such medications prior to TAVR. Proton pump inhibitors and histamine antagonists were often utilized for GI prophylaxis but were not a part of a routine post-procedural TAVR order set. Data regarding medication administration was extracted from a computerized system that records each encounter for medication administration based on a unique barcode that the administering nurse scans into the medication delivery system.

Medical record numbers were extracted from an ongoing database of patients undergoing TAVR at UCDMC over this time. Of these, patients with incomplete and/or inadequate medical records and inmates were excluded. Data extracted included, patient demographics, hemoglobin, MCV, creatinine, platelets and INR on admission, antithrombotic therapy including vitamin $\mathrm{K}$ antagonists or novel oral anticoagulants (NOACs) and antiplatelet therapies pre-procedure, colon cancer screening status, history of chronic kidney disease and chronic liver disease, history of GI disease and GI prophylaxis. Patients who developed GI bleeding were identified from review of medical records. GIB was defined as clinical evidence of upper or lower GIB such as hematemesis, melena, and hematochezia or hemoglobin drop of $2 \mathrm{~g} / \mathrm{dl}$ without any other obvious non-GI cause. The incidence of GIB was calculated by dividing the number of all consecutive cases in the specified period by the number of GIB (both upper and lower) cases. In patients who underwent endoscopy, data regarding etiology and location of the bleeding lesions and need for endoscopic treatment was extracted from the procedure note.

\section{Statistical Analysis}

Descriptive data was reported as percentages, means and standard deviation (with range when appropriate). For comparison of the clinical variables in the 2 groups (GIB vs. non-GIB), we used t-test for continuous variables and Fisher exact test for categorical variables. Due to severe imbalance in the sample size in the two groups, asymptotic properties may not be well met (e.g., interpretation of p-value). Univariate regression analysis was performed to estimate odds ratio between risk factors and GIB, and due to rare assumption, odds ratio may approximate risk ratio reasonably well.

\section{Results}

Of 508 records reviewed, 489 met the inclusion criteria. Mean age was $81.6 \pm 8.2$ years (range 27 to 98 ). Women and men were represented equally in our study. Baseline characteristics of patients are summarized in (Table 1). Mean baseline hemoglobin level in the group with GI bleeding was lower than the group who did not have post procedural GIB (10.6 g/dl vs $12.0 \mathrm{~g} / \mathrm{dl}, \mathrm{p}=0.04)$. Dual antiplatelet therapy (DAPT) was 
used in 396 (81.0\%) patients. Anticoagulation (mainly with warfarin) was used in 155 (31.7\%) patients. Triple therapy with DAPT and anticoagulation was used in 91 patients (18.6\%). Gastric prophylaxis with either PPIs or $\mathrm{H} 2$ receptor blockers was used in 336 (68.7\%).

Incidence of GIB was 1.4\% (7/489). Gastroenterology consultation was requested for hematemesis in three patients, melena in two patients, hematochezia in one patient and acute drop in hemoglobin in one patient. Endoscopy was recommended by the consulting gastroenterologist in all patients; however, two refused to undergo endoscopy. Three patients underwent upper endoscopy, one patient underwent both upper endoscopy and colonoscopy and one patient underwent upper endoscopy, colonoscopy and video capsule endoscopy of the small intestine. After appropriate work up, five cases of upper GIB, one case of small intestinal bleeding and one case of lower GIB were identified. Angiodysplasia was seen in three patients (one in stomach, one in jejunum and one in cecum) and esophagitis in two patients. Endoscopic treatments in the form of argon plasma coagulation were rendered in three patients; one patient with a deep gastroesophageal junction ulcer and another patient with severe esophagitis were not candidates for endoscopic hemostatic intervention. No patients needed interventional radiology or surgical intervention for control of bleeding. Four patients needed packed red blood cell transfusion. Mean length of hospitalization was 6.1 days. It was significantly longer in patients with GIB compared to those without GIB (8.7 vs 6.0 days, $p=0.026$ ). Complete characteristics and variables specific to the gastrointestinal bleeding group for the above studied patients admitted for TAVR are displayed in (Table 2).

Using univariate logistic regression, we analyzed the effect of age, baseline hemoglobin, DAPT, triple anticoagulation, gastric prophylaxis, history of GIB, CKD and chronic liver disease on occurrence of GIB after TAVR. Lower baseline hemoglobin ( $1 \mathrm{~g} / \mathrm{dl}$ decrease) was associated with increased odds of GIB (OR 1.49, $95 \% \mathrm{Cl}$ 0.998-2.24, $\mathrm{p}=0.051$ ).

\section{Discussion}

Although early (within 30 days) and late (after 30 days) bleeding complications of TAVR have been reported on [7-9], there is limited data regarding the incidence of GIB during the immediate post procedural period. We report a single center experience of 489 patients undergoing TAVR at a tertiary care hospital. The overall rate of both upper and lower GIB bleeding was $1.4 \%$ in our cohort. Similarly, a study on 841 patients undergoing TAVR reported an incidence of $2 \%$ for upper GIB in the immediate post TAVR period [10]. For comparison, the rate of GIB within 30 days and after 30 days of TAVR have were reported to be $2.41 \%$ and $5.4 \%$, respectively $(7,8)$

The most common culprit lesion found during endoscopy in our cohort was angiodysplasia occurring in $0.6 \%$ of patients. The incidence of GIB presumed due to small intestinal angiodysplasia in patients with untreated AS has been reported at $0.9 \%$ in one study [11]. The association between severe AS and GIB is classically represented in Hyde syndrome. First described by Edward Hyde in 1958 [12], the pathogenesis of this syndrome is thought to involve an acquired von Willebrand Factor (VWF) deficiency due to shear stress from the stenotic aortic valve and formation of angiodysplasia in the gastrointestinal mucosa due to 
relative ischemia [13]. In our cohort, all patients with angiodysplasia as their source of GIB were successfully treated with argon plasma coagulation.

As mentioned earlier, patients who undergo TAVR are at risk for GIB due to multiple reasons and it may prove useful to identify preprocedural risk factors that could be modified. In our cohort, the only factor that predicted a risk of bleeding was low hemoglobin at baseline. We hypothesize that patients who had low hemoglobin prior to TAVR likely had preexisting lesions, such as angiodysplasia, which had caused anemia through ongoing low-level bleeding. Subsequently, these underlying lesions were unmasked and resulted in GIB in the post procedure period due to factors related to the procedure itself or related pharmacotherapy such as anticoagulation or triple therapy.

In our cohort, DAPT and triple therapy with concomitant DAPT and anticoagulation were not found to be associated with increased likelihood of GIB in the immediate postop period $(p>0.1)$. The existing literature on the correlation between DAPT, anticoagulation and risk of bleeding is conflicting. Some studies have reported that patients on postprocedural triple antithrombotic therapy were at the highest risk for severe upper GIB. More specifically, these patients were found to be at a $>10$-fold higher risk of upper GIB when compared to patients not on triple antithrombotic therapy [10]. However, a meta-analysis of nine studies comparing DAPT with either aspirin monotherapy or monoantiplatelet therapy (MAPT) plus anticoagulation did not show an increased risk of major bleeding in the DAPT group although GI bleeding was not specifically reported in the study $(\mathrm{OR}, 1.69 ; 95 \% \mathrm{Cl}, 0.86-3.31 ; \mathrm{P}=0.13)$ [14]. In another meta-analysis of 410 patients, the relative risk of major bleeding in patients who were treated with DAPT compared to MAPT was $41 \%\left(95 \% \mathrm{Cl}, 0.25-0.69, \mathrm{Z}=3.41, \mathrm{P}=0.0006, \mathrm{I}^{2}=33 \%\right)$. After removing a single study which was the major contributor to increased risk in this review, no statistically significant difference was observed between groups in terms of major bleeding ( $\mathrm{RR}=0.6495 \% \mathrm{Cl}, 0.34-1.22, \mathrm{P}=0.18)$ [15]. One of the factors that could explain for a lack of increased risk of bleeding with either DAPT or concomitant DAPT and anticoagulation in our study may be the short time frame in which patients were analyzed in this study which did not allow for these medications to reach therapeutic levels. It is also possible that patients may have been discharged home and subsequently suffered a significant GIB that was not captured within the immediate index hospitalization. Finally, this finding may also be due to a relatively low incidence of the primary endpoint (GIB) and it is possible that our study was not adequately powered to detect a small difference.

GI prophylaxis, with either proton pump inhibitor or histamine antagonist, was given post-procedurally to $69 \%$ (336/489) of patients undergoing TAVR. The rate of GIB did not differ between those who did and did not receive GI prophylaxis. Similar results have been reported in a large randomized controlled trial of 222 patients who were assigned to either aspirin alone or aspirin plus clopidogrel. All GIB events $(5 / 111)$ occurred in patients who were on proton pump inhibitors [16].As stated above, angiodysplasia was the most common culprit lesion found during endoscopy in our cohort which is a condition not responsive to the therapeutic effects of acid suppression. Given the above findings, future studies should continue to explore the utility and safety of prescribing post-procedural GI prophylaxis, specifically PPIs, to decrease the risk of GIB, which has historically been left to the physicians' judgment. PPIs cause side effects such as acute interstitial nephritis, clostridium difficile infection, pneumonia, spontaneous bacterial peritonitis, electrolyte 
and malabsorption abnormalities and osteoporosis, to name a few, and have also been implicated in drugdrug interactions [17].

One of the strengths of our study is the relatively large sample size over a long period of time. Another strength of this study is the means by which medication administration data was collected and the accuracy of this data. In our study medication administration date, time, dose, and route was extracted by reviewing data which was populated during each nursing medication administration encounter thereby minimizing any errors which might have caused if medication data was gathered through review of physician notes or medication administration records. To the best of our knowledge our study is also the first to report the rate of both upper and lower GI bleeding during index hospitalization for TAVR.

Our study is limited by its retrospective examination of the TAVR database at a tertiary center and the low rate of the outcome of interest which could cause lack of statistical significance. Lastly, our study focused on the events happening during the index hospitalization and therefore any bleeding occurring later would have been missed. The prognostic significance of immediate postop bleeding remains uncertain. Therefore, future studies are needed to investigate any association between bleeding during index hospitalization and early and late bleeding. Future studies should be performed to assess applicability and possible benefits of performing preoperative workup with endoscopy prior to TAVR procedure in patients with baseline anemia. Lastly, the necessity of the largely accepted PPI therapy for GI prophylaxis should be further investigated and validated.

\section{CONCLUSIONS}

In conclusion, we demonstrate gastrointestinal bleeding is rare immediately post-transcatheter aortic valve replacement. Angiodysplasia was the most common etiology in our series of 482 elderly patients, $23 \%$ of whom had advanced chronic kidney disease. When indicated, endoscopy was a safe and successful method in achieving hemostasis in our patient population. Baseline anemia is a predictor of gastrointestinal bleeding in this setting.

\section{Abbreviations}

AS, Aortic stenosis; CKD, chronic kidney disease; DAPT, Dual antiplatelet therapy; GIB, Gastrointestinal bleeding; Hgb, hemoglobin; MAPT, monoantiplatelet therapy; NOACs, novel oral anticoagulants; PPI, proton pump inhibitor; TAVR, transcatheter aortic valve replacement; UCDMC, UC David Medical Center; vWF, von Willebrand Factor.

\section{Declarations}

Ethics approval and consent to participate: For this type of study, formal consent is not required. This was retrospective review of de-identified medical records and other data. A waiver of consent was granted by institutional IRB due to minimal risk nature of the study. 
Availability of data and material: All data generated and/or analyzed during this study are included as supplementary information files.

Competing interests: The authors declare that they have no competing interests.

Funding: The project described was partly supported (analysis and interpretation of data) by the National Center for Advancing Translational Sciences, National Institutes of Health, through grant number UL1 TR001860.

Authors' contributions: TS (acquisition of data; analysis and interpretation of data; drafting of the manuscript), AE (study concept and design; analysis and interpretation of data, drafting of the manuscript), TW (acquisition of data), HB (analysis and interpretation of data; critical revision of the manuscript for important intellectual content; statistical analysis), BS (procurement of data base and acquisition of data, drafting of the manuscript), JC (procurement of data base and acquisition of data, drafting of the manuscript), GW (procurement of data base and acquisition of data, drafting of the manuscript), JS (procurement of data base and acquisition of data, drafting of the manuscript), WB (procurement of data base and acquisition of data, drafting of the manuscript), ST (study concept and design; analysis and interpretation of data; drafting of the manuscript; critical revision of the manuscript for important intellectual content; obtained funding; administrative, technical, or material support ; study supervision). All authors read and approved the final manuscript.

Acknowledgements: Not applicable.

\section{References}

1. Bhatia, N., et al., Aortic valve disease in the older adult. J Geriatr Cardiol, 2016. 13(12): p. 941-944.

2. Eveborn, G.W., et al., The evolving epidemiology of valvular aortic stenosis. the Tromso study. Heart, 2013. 99(6): p. 396-400.

3. Nkomo, V.T., et al., Burden of valvular heart diseases: a population-based study. Lancet, 2006. 368(9540):

p. 1005-11.

4. Grover, F.L., et al., 2016 Annual Report of The Society of Thoracic Surgeons/American College of Cardiology Transcatheter Valve Therapy Registry. J Am Coll Cardiol, 2017. 69(10): p. 1215-1230.

5. Messenger, J.C., Trends in United States TAVR Practice. An update on where TAVR is going as the technology matures in the United States. Cardiac Interventions Today, 2018. 12(March/April 2018): p. 46-50.

6. Bossone, E., et al., Valve surgery in octogenarians: in-hospital and long-term outcomes. Can J Cardiol, 2007. 23(3): p. 223-7. 
7. Genereux, P., et al., Incidence, predictors, and prognostic impact of late bleeding complications after transcatheter aortic valve replacement. J Am Coll Cardiol, 2014. 64(24): p. 2605-2615.

8. Nombela-Franco, L., et al., Incidence, Causes, and Predictors of Early ( $</=30$ Days) and Late Unplanned Hospital Readmissions After Transcatheter Aortic Valve Replacement. JACC Cardiovasc Interv, 2015. 8(13): p. $1748-57$.

9. Piccolo, R., et al., Frequency, Timing, and Impact of Access-Site and Non-Access-Site Bleeding on Mortality Among Patients Undergoing Transcatheter Aortic Valve Replacement. JACC Cardiovasc Interv, 2017. 10(14): p. 1436-1446.

10. Stanger, D.E., et al., Upper gastrointestinal bleeding following transcatheter aortic valve replacement: $A$ retrospective analysis. Catheter Cardiovasc Interv, 2017. 90(2): p. E53-E61.

11. Pate, G.E. and A. Mulligan, An epidemiological study of Heyde's syndrome: an association between aortic stenosis and gastrointestinal bleeding. J Heart Valve Dis, 2004. 13(5): p. 713-6.

12. Heyde, E.J., Gastrointestinal bleeding in aortic stenosis. N Engl J Med, 1958. 259: p. 196.

13. Vincentelli, A., et al., Acquired von Willebrand syndrome in aortic stenosis. N Engl J Med, 2003. 349(4): p. 343-9.

14. Verdoia, M., et al., Dual Versus Single Antiplatelet Regimen With or Without Anticoagulation in Transcatheter Aortic Valve Replacement: Indirect Comparison and Meta-analysis. Rev Esp Cardiol (Engl Ed), 2018. 71(4): p. 257-266.

15. Aryal, M.R., et al., Dual versus single antiplatelet therapy in patients undergoing transcatheter aortic valve replacement: a systematic review and meta-analysis. Heart Lung Circ, 2015. 24(2): p. 185-92.

16. Rodes-Cabau, J., et al., Aspirin Versus Aspirin Plus Clopidogrel as Antithrombotic Treatment Following Transcatheter Aortic Valve Replacement With a Balloon-Expandable Valve: The ARTE (Aspirin Versus Aspirin + Clopidogrel Following Transcatheter Aortic Valve Implantation) Randomized Clinical Trial. JACC Cardiovasc Interv, 2017. 10(13): p. 1357-1365.

17. Benmassaoud, A., E.G. McDonald, and T.C. Lee, Potential harms of proton pump inhibitor therapy: rare adverse effects of commonly used drugs. CMAJ, 2016. 188(9): p. 657-662.

\section{Tables}

Table 1: Baseline Variables of Non Gastrointestinal Bleeding Group and Gastrointestinal Bleeding Group in Patients Admitted for TAVR to UC Davis Health System, January 2012 to June 2017. 


\begin{tabular}{|l|c|c|c|}
\hline \multirow{2}{*}{} & \multicolumn{2}{|c|}{ Mean (SD) for continuous variables or } & \\
& $\mathbf{n}(\%)$ for binary variables & \\
\hline Variables & No GIB (n=482) & All GIB (n=7) & P-value \\
\hline Age (years) & $81.6(8.2$, range: $27-98)$ & $81.6(10.8$, range: $68-93)$ & 0.98 \\
\hline Male & $239(48.9 \%)$ & $3(0.6 \%)$ & 1 \\
\hline HGB (g/dL) & $12.0(1.8)$ & $10.6(2.1)$ & 0.049 \\
\hline MCV (fL) & $91.6(6.7)$ & $93.9(4.8)$ & 0.35 \\
\hline Platelet count $\left(10^{3} / \mu \mathrm{L}\right)$ & $197(68)$ & $188(48)$ & 0.72 \\
\hline INR & $1.1(0.2) \dagger$ & $1.2(0.4)$ & 0.60 \\
\hline Serum Creatinine $(\mathrm{mg} / \mathrm{dl})$ & $1.4(1.0)$ & $1.6(1.0)$ & 0.62 \\
\hline Dual antiplatelet therapy & $391(80.0 \%)$ & $5(1.0 \%)$ & 0.62 \\
\hline Anticoagulation & $151(30.9 \%)$ & $4(0.8 \%)$ & 0.22 \\
\hline Triple therapy & $88(18.0 \%)$ & $3(0.6 \%)$ & 0.12 \\
\hline Gastric prophylaxis & $329(67.3 \%)$ & $7(1.4 \%)$ & 0.1 \\
\hline Chronic liver disease & $19(3.9 \%)$ & $0(0 \%)$ & 1 \\
\hline Chronic kidney disease & $111(22.7 \%)$ & $2(0.4 \%)$ & 0.66 \\
\hline History of GIB & $165(33.7 \%)$ & $1(0.2 \%)$ & 0.43 \\
\hline History of colonoscopy* & $95(29.8 \%)$ & $6(1.9 \%)$ & 0.0009 \\
\hline
\end{tabular}

(\%) of total population $(\mathrm{n}=489)$

*Data available for 319 patients only; †data available for 480 patients.

P-values for continuous variables are from Wilcoxon Test and those for binary variables are from t-test.

DAPT: Dual antiplatelet therapy; GIB: Gastrointestinal bleeding; INR: International normalized ratio;

HGB: Hemoglobin; MCV: Mean corpuscular volume; SD: Standard deviation.

Table 2: Patient Characteristics and Endoscopic Variables in those with GI Bleeding Post TAVR, January 2012 to June 2017. 


\begin{tabular}{|c|c|c|c|c|c|c|c|c|c|c|}
\hline Age & Sex & HGB & MCV & DAPT & $\begin{array}{c}\text { Gastric } \\
\text { ppx }\end{array}$ & CKD & $\begin{array}{l}\text { History } \\
\text { of GIB }\end{array}$ & Reason & Lesion & Treatment \\
\hline 72 & Male & 9.2 & 95.7 & No & Yes & Yes & Yes & Acute anemia & $\begin{array}{c}\text { Jejunal } \\
\text { angiodysplasia }\end{array}$ & APC \\
\hline 71 & Female & 9.2 & 91.9 & No & Yes & No & No & Hematemesis & Refused EGD & N/A \\
\hline 87 & Female & 12.1 & 94.8 & No & No & No & No & Hematemesis & $\begin{array}{l}3 \text { cm deep } \\
\text { GE junction } \\
\text { ulcer }\end{array}$ & $\begin{array}{c}\text { Not } \\
\text { amenable to } \\
\text { endotherapy }\end{array}$ \\
\hline 68 & Male & 7.7 & 91.7 & No & No & Yes & No & Hematemesis & $\begin{array}{c}4-5 \\
\text { subcentimeter } \\
\text { Gastric } \\
\text { angiodysplasias }\end{array}$ & APC \\
\hline 92 & Male & 13.8 & 94.5 & No & Yes & No & No & Hematochezia & Refused EGD & $\mathrm{N} / \mathrm{A}$ \\
\hline 93 & Female & 11.8 & 102.5 & No & No & No & No & Melena & Esophagitis & $\begin{array}{c}\text { No } \\
\text { endotherapy } \\
\text { indicated }\end{array}$ \\
\hline 88 & Female & 10.5 & 86.5 & No & No & No & No & Melena & $\begin{array}{c}7 \mathrm{~mm} \\
\text { Cecal } \\
\text { angiodysplasia }\end{array}$ & $\mathrm{APC}$ \\
\hline
\end{tabular}

APC: Argon plasma coagulation; CKD: Chronic kidney disease; DAPT: Dual antiplatelet therapy; EGD:

Esophagogastroduodenoscopy; GE: Gastroesophageal; GIB: Gastrointestinal bleeding; HGB: Hemoglobin; MCV:

Mean corpuscular volume; ppx: Prophylaxis.

\section{Supplementary Files}

This is a list of supplementary files associated with this preprint. Click to download.

- supplement1.xlsx 